\title{
Dilepton Forward-Backward Asymmetry and electroweak mixing angle at ATLAS and CMS
}

\author{
Jiyeon Han for the ATLAS and CMS collaborations* ${ }^{* \dagger}$ \\ University of Rochester \\ E-mail: jyhan@fnal.gov
}

\begin{abstract}
We present measurements of the forward-backward asymmetry in Drell-Yan dilepton (ee and $\mu \mu$ ) events with the data sample of pp collisions at $\sqrt{s}=7 \mathrm{TeV}$ and $8 \mathrm{TeV}$ collected by ATLAS and CMS detector. At CMS, the measurements are extended up to the boson rapidity, $|y|=5$, by including forward electrons. At ATLAS, the measured forward-backward asymmetry is used to extract the effective electroweak mixing angle, $\sin ^{2} \theta_{\text {eff }}^{\text {lept }}$. The measured $\sin ^{2} \theta_{\text {eff }}^{\text {lept }}$ from combining all channels is $0.2308 \pm 0.0005$ (stat.) \pm 0.0006 (syst.) \pm 0.0009 (PDF).
\end{abstract}

38th International Conference on High Energy Physics

3-10 August 2016

Chicago, USA

* Speaker.

${ }^{\dagger}$ A footnote may follow. 


\section{Introduction}

The forward-backward asymmetry $\left(A_{F B}\right)$ of Drell-Yan process originates from the interference of vector and axial-vector couplings of electroweak bosons to fermions. The forward-backward asymmetry is defined as

$$
A_{F B}=\frac{\sigma_{F}-\sigma_{B}}{\sigma_{F}+\sigma_{B}}
$$

where $\sigma_{F}\left(\sigma_{B}\right)$ is the total cross section for the forward (backward) events. The forward (backward) events are defined by $\cos \theta>0(\cos \theta<0)$ where $\theta$ is the emission angle of the negatively charged lepton relative to the quark momentum in the rest frame of the dilepton system. $A_{F B}$ has a strong dependence on the dilepton mass (M). It is close to zero near $\mathrm{Z}$ boson mass peak, whereas it is large and negative (positive) at low (high) dilepton mass. The $A_{F B}$ parameter as a function of dilepton mass is sensitive to the electroweak mixing angle $\sin ^{2} \theta_{W}$, and has been used to extract the effective weak mixing angle.

To reduce the uncertainties due to the transverse momentum $\left(p_{T}\right)$ of the incoming quarks, $A_{F B}$ is calculated in the Collins-Soper (CS) frame [1]. In pp collision, the quark $(q)$ and anti-quark $(\bar{q})$ direction is not known. However, the $\mathrm{Z}$ boson is boosted into the quark direction on average since the quark carries more momentum than the anti-quark as the anti-quark must originate from the parton sea. Therefore, the quark direction (positive z-axis) is determined based on the rapidity direction in the measurement. Since the ambiguity of determining the quark direction increases at low rapidity, the $A_{F B}$ measurement in pp collision has a strong rapidity dependence. $A_{F B}$ is more pronounced at large rapidity due to the better identification of quark direction. Here, we present the latest $A_{F B}$ measurements as a function of dilepton invariant mass in ATLAS and CMS.

\section{Data and event selection}

The analysis is performed using pp collision data collected with ATLAS and CMS detector. $[2,3]$ The ATLAS data corresponds to the integrated luminosity of 4.8 (4.6) $\mathrm{fb}^{-1}$ at $\sqrt{s}=7 \mathrm{TeV}$ for dielectron (dimuon) candidates. A single lepton $(e$ or $\mu)$ trigger is used with $E_{T}(e)>20$ or 22 $\mathrm{GeV}$ depending on the instantaneous luminosity and $p_{T}(\mu)>18 \mathrm{GeV}$. Electrons are categorized to central (C) and forward (F) electron based on the pseudorapidity $(\eta)$ range, $|\eta|<2.47$ for central electrons and $2.5<|\eta|<4.9$ for forward electrons. All electrons are required to have $E_{T}>25 \mathrm{GeV}$. Di-electrons have two event topologies. Here, $\mathrm{CC}$ events have two central electrons which are required to be oppositely charged electrons and pass 'medium' identification selection. The CF events have one 'tight' central electron and one 'medium' forward electron candidate. An isolation energy requirement $\left(E_{i s o}<5 \mathrm{GeV}\right)$ is applied for the central electron in $\mathrm{CF}$ events to reduce the background. In the muon channel, muons are required to have $p_{T}>20 \mathrm{GeV}$ and $|\eta|<2.4$. The identification selection of muon is based on quality requirements for the sub-detector components and a fractional tracker isolation requirement $\left(\sum p_{T}^{\text {track }} / p_{T}^{\mu}<0.1\right)$.

The CMS data corresponds to an integrated luminosity of $19.7 \mathrm{fb}^{-1}$ at $\sqrt{s}=8 \mathrm{TeV}$ for both dielectron and dimuon candidates. A dilepton $\left(e\right.$ or $\mu$ ) trigger with $E_{T}(e)$ or $p_{T}(\mu)>17$ and $8 \mathrm{GeV}$ with $|\eta|<2.4$ is required for the leading and sub-leading lepton. For forward dielectron events, a single electron trigger with $E_{T}(e)>27 \mathrm{GeV}$ and $|\eta|<2.4$ is required. Dielectron candidates 
in CMS are selected in a similar way as ATLAS. Central electrons are selected with $E_{T}>20$ or $30 \mathrm{GeV}$ and $|\eta|<2.4$ and are required to pass electron identification criteria. Central electrons are required to have a matched track and pass a fractional particle flow isolation requirement. Forward electrons are selected with $E_{T}>20 \mathrm{GeV}$ and $3<|\eta|<5$ requiring the selection criteria. The selections of the forward electrons are optimized based on isolation variables. CC events are selected with two central electrons with $E_{T}>20 \mathrm{GeV}$ and are required to have opposite charges. $\mathrm{CF}$ events are selected with one central electron $\left(E_{T}>30 \mathrm{GeV}\right)$ and one forward electron $\left(E_{T}>20\right.$ $\mathrm{GeV})$ and both electrons are required to be on the same side of detector $\left(\eta_{1} \times \eta_{2}>0\right)$ to reduce the dijet background contribution. Muon candidates are required to have $p_{T}>20 \mathrm{GeV}$ and $|\eta|<2.4$ and pass muon identification criteria and a fractional tracker isolation requirement $\left(\sum p_{T}^{\text {track }} / p_{T}^{\mu}<\right.$ $0.1)$.

\section{Simulations}

Simulated $Z / \gamma^{*} \rightarrow \mu \mu$ and $Z / \gamma^{*} \rightarrow e e$ signal samples are generated differently in CMS and ATLAS. The ATLAS measurement uses the PYTHIAv6.4 generator [4] with MSTW2008(LO) parton distribution functions (PDFs) [5] with $\sin \theta^{2} \theta_{\text {eff }}^{\text {lept }}=0.232$. In CMS the POWHEG generator [6] with CT10 (NLO) PDFs [7] with $\sin \theta^{2} \theta_{\text {eff }}^{\text {lept }}=0.2312$ is used. For the background samples, both measurements use various generator programs depending on the physics process. All samples include a full simulation of the detector based on GEANT4 [8]. In addition, the MC simulations are tuned to describe the data including pileup distribution, efficiencies for trigger, identification, and reconstruction, and lepton energy/momentum scales and resolutions. More details for the simulation samples are described in [11, 10].

\section{Background estimation}

The major backgrounds for the Drell-Yan process are $Z / \gamma^{*} \rightarrow \tau \tau$ at low mass and $t \bar{t}$ in the high mass region. In addition, the multi-jet background is one of the dominant sources in the dielectron channel. In ATLAS $Z / \gamma^{*} \rightarrow \tau \tau$, $t \bar{t}$, and diboson $(W W / W Z / Z Z)$ processes are estimated using MC simulations. The multi-jet background is estimated using a data-driven method which uses a control region selected by applying reverse lepton identification criteria. In CMS, the multi-jet background is measured using same charge events for both dielectron (CC) and dimuon channels. For CF dielectron events a fitting method is used. The $Z / \gamma^{*} \rightarrow \tau \tau, t \bar{t}$, $\mathrm{W}+\mathrm{jets}$, and tW processes are estimated using the $e \mu$ method for the muon channel. All other remaining backgrounds are estimated using MC simulations.

The dilepton invariant mass distribution of selected events for data and MC simulation (including background contributions) are shown in Fig. 1 for ATLAS, and 2 for CMS, respectively.

\section{Measurement of $A_{F B}$}

The measured $A_{F B}$ for reconstructed events $\left(A_{F B}^{r a w}\right)$ is defined as

$$
A_{F B}=\frac{N_{\cos \theta_{C S} \geq 0}-N_{\cos \theta_{C S}<0}}{N_{\cos \theta_{C S} \geq 0}+N_{\cos \theta_{C S}<0}}
$$



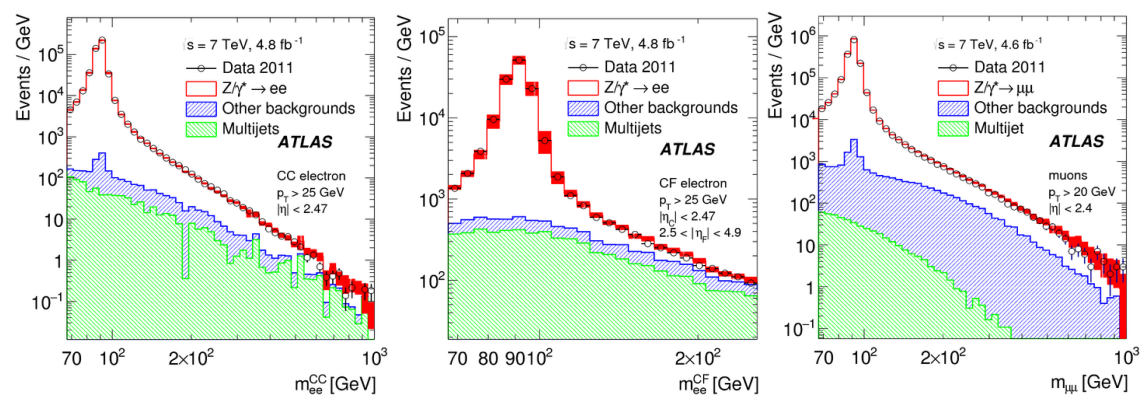

Figure 1: Dilepton invariant mass distributions for CC electrons (left), $\mathrm{CF}$ electrons (middle), and dimuon (right) events in ATLAS, for $\sqrt{s}=7 \mathrm{TeV}$ are compared for data and MC simulations (including background contributions).
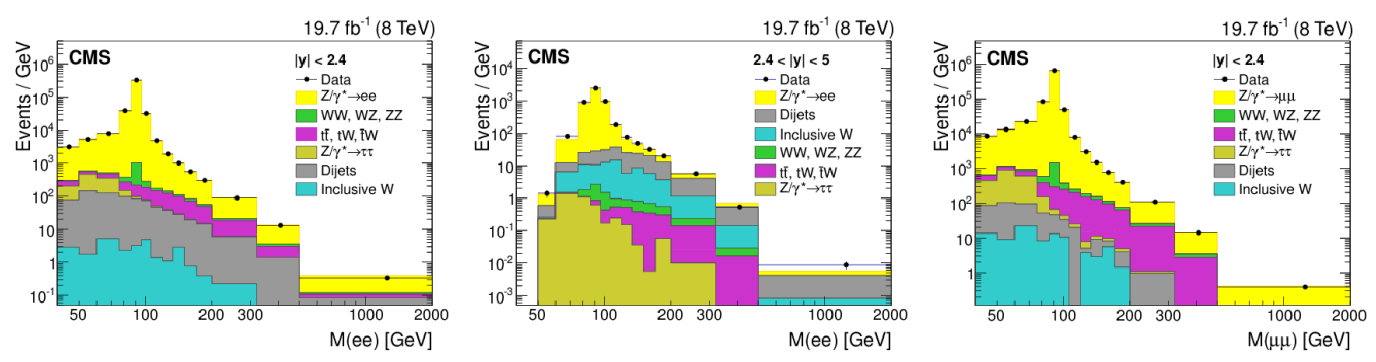

Figure 2: Dilepton invariant mass distributions for CC electrons (left), CF electrons (middle), and dimuons (right) events in CMS at $\sqrt{s}=8 \mathrm{TeV}$ are compared for data and MC simulations (including background contributions.

ATLAS collaboration measures $A_{F B}$ in bins of dilepton invariant mass for three events categories, ee(CC), ee(CF), and $\mu \mu$ events. The ATLAS measurement is further corrected for dilution effects which originate from the mis-identification of the quark direction. A correction factor for the effect of dilution is estimated using PYTHIA signal MC samples.

The CMS collaboration measures $A_{F B}$ in in bins of dilepton invariant mass for five dilepton rapidity bins, $|y|<1,1<|y|<1.25,1.25<|y|<1.5,1.5<|y|<2.4$, and $2.4<|y|<5$. Subsequently, the $A_{F B}$ measurements are corrected for the detector resolution, acceptance, efficiency, and the effect of final-state QED radiation (FSR) using an iterative unfolding method based on Bayes' theorem [9]. Fig. 3 show the unfolded $A_{F B}$ as a function of dilepton invariant mass and rapidity measured in CMS. The unfolded $A_{F B}$ is combined for both $e e$ and $\mu \mu$ events up to $|y|=2.4$. The highest rapidity bin, $2.4<|y|<5$, includes only CF electron events. The unfolded $A_{F B}$ is in good agreement with the POWHEG (NLO) prediction with $\sin ^{2} \theta_{\text {eff }}^{\text {lept }}=0.2312$ for all rapidity regions. [10]

The unfolded $A_{F B}$ in the ATLAS measurement at the the Born level including correction of dilution is shown in Fig. 4 for ee(CC), ee(CF), and $\mu \mu$ events, respectively. The ATLAS measurement is compared with the PYTHIA prediction with $\sin ^{2} \theta_{\text {eff }}^{\text {lept }}=0.232$. The unfolded $A_{F B}$ agrees with the PYTHIA prediction in general. However, some deviation is observed at high mass in the muon channel. [11]

The major systematic uncertainties in the $A_{F B}$ measurement originate from the lepton energy/momentum scale and resolution, unfolding procedure, and background estimation. 

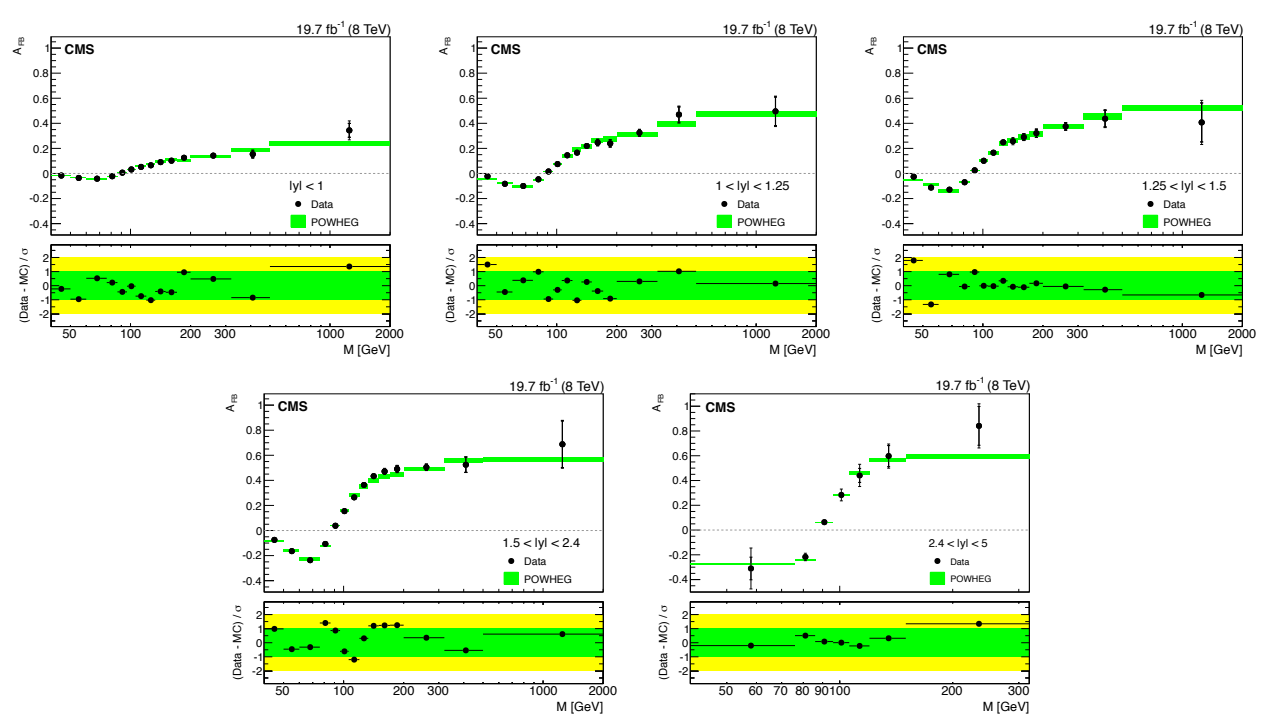

Figure 3: The unfolded forward-backward asymmetry measured in CMS as a function of the dilepton invariant mass for the five rapidity bins.
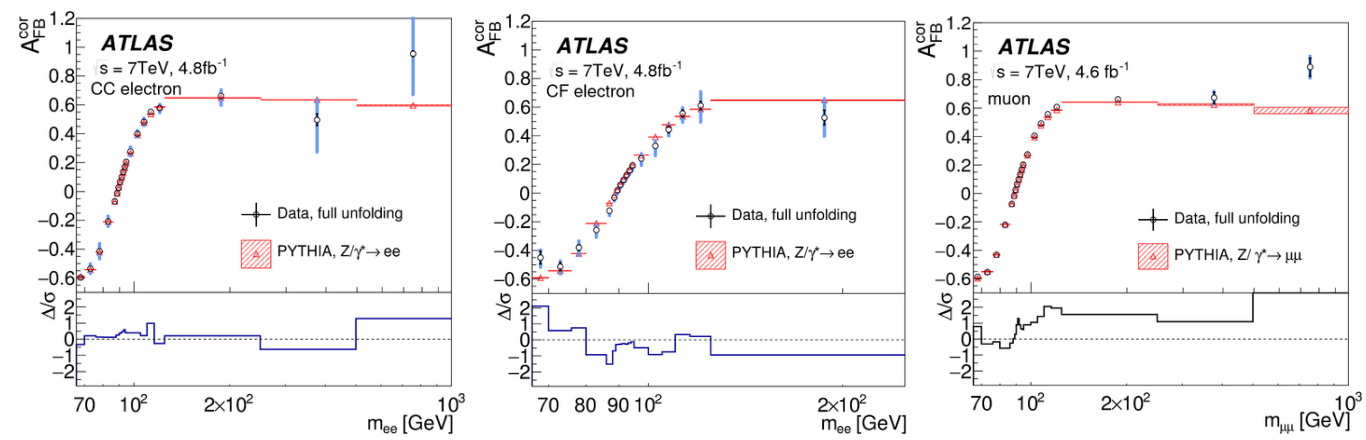

Figure 4: The unfolded forward-backward asymmetry measured in ATLAS as a function of the dilepton invariant mass for $\mathrm{CC}$ electrons (left), $\mathrm{CF}$ electrons (middle), and dimuon (right) events.

\section{Measurement of $\sin ^{2} \theta_{\text {eff }}^{\text {lept }}$}

ATLAS collaboration extracts the effective weak mixing angle from the measured $A_{F B}^{\text {raw }}$. The extraction is done using a template method with a PYTHIA MC simulation. Templates for $A_{F B}$ are constructed by varying $\sin ^{2} \theta_{\text {eff }}^{\text {lept }}$ in the range $0.218 \leq \sin ^{2} \theta_{\text {eff }}^{\text {lept }} \leq 0.236$. Weights for each $\sin ^{2} \theta_{e f f}^{\text {lept }}$ value are calculated at the generator level, in bins of dilepton invariant mass and $\cos \theta_{C S}$. The $A_{F B}^{\text {raw }}$ values from the re-weighted MC samples are compared with data and $\chi^{2}$ test between data and templates over the dilepton invariant mass range of 70 to $250 \mathrm{GeV}$ is used to extract $\sin ^{2} \theta_{\text {eff }}^{\text {lept }}$. Table 1 summarizes the extracted $\sin ^{2} \theta_{\text {eff }}^{\text {lept }}$ in each of the three channels (CC electron, $\mathrm{CF}$ electron, and muon). The dominant systematic uncertainty of the $\sin ^{2} \theta_{\text {eff }}^{\text {lept }}$ measurement is the PDF uncertainty. [11] 


\begin{tabular}{l|c}
\hline \hline & $\sin ^{2} \theta_{\text {eff }}^{\text {lept }}$ \\
\hline \hline CC electron & $0.2302 \pm 0.0009$ (stat.) \pm 0.0008 (syst.) $\pm 0.0010(\mathrm{PDF})=0.2302 \pm 0.0016$ \\
CF electron & $0.2312 \pm 0.0007$ (stat.) \pm 0.0008 (syst.) $\pm 0.0010(\mathrm{PDF})=0.2312 \pm 0.0014$ \\
Muon & $0.2307 \pm 0.0009$ (stat.) \pm 0.0008 (syst.) \pm 0.0009 (PDF) $=0.2307 \pm 0.0015$ \\
El. combined & $0.2308 \pm 0.0006$ (stat.) \pm 0.0007 (syst.) $\pm 0.0010(\mathrm{PDF})=0.2308 \pm 0.0013$ \\
\hline Combined & $0.2308 \pm 0.0005$ (stat.) \pm 0.0006 (syst.) \pm 0.0009 (PDF) $=0.2308 \pm 0.0012$ \\
\hline
\end{tabular}

Table 1: ATALAS measurements of the effective weak mixing angle in three channels, CC electrons, $\mathrm{CF}$ electrons and dimuons. Also shown is statistical combination of both electron channels and all three channels.

\section{Conclusion}

The forward-backward asymmetry in Drell-Yan events is measured at ATLAS $\left(\int L=4.8 f b^{-1}\right.$ at $\sqrt{s}=7 \mathrm{TeV})$ and $\mathrm{CMS}\left(\int L=19.7 f b^{-1}\right.$ at $\left.\sqrt{s}=8 \mathrm{TeV}\right)$ with Run I data. The measurements are in good agreement with PYTHIA and POWHEG theory predictions. The $A_{F B}$ measurements in ATLAS are used to extract the effective mixing angle. The measured $\sin ^{2} \theta_{\text {eff }}^{\text {lept }}$ from combining all channels in ATLAS is $0.2308 \pm 0.0005$ (stat.) \pm 0.0006 (syst.) \pm 0.0009 (PDF).

\section{References}

[1] J.C. Collins and D.E. Soper, "Angular Distribution of Dileptons in High-Energy Hadron Collisions", Phys. Rev. D 16 (1977) 2219.

[2] ATLAS Collaboration, "The ATLAS experiment at the CERN LHC", JINST 3 (2008) S08003.

[3] CMS Collaboration, "The CMS experiment at the CERN LHC", JINST 3 (2008) S08004.

[4] T. Sjöstrand, S. Mrenna, and P. Skands, "PYTHIA 6.4 physics and manual", JHEP 05 (2006) 026.

[5] A. Martin et al., "Parton distributions for the LHC", Eur. Phys. J. C 63 (2009) 189.

[6] S. Alioli, P. Nason, C. Oleari, and E. Re, "NLO vector-boson production matched with shower in POWHEG", JHEP 07 (2008) 060

[7] H.-L. Lai et al., "New parton distributions for collider physics", Phys. Rev. D 82 (2010) 074024.

[8] GEANT4 Collaboration, "GEANT4?a simulation toolkit", Nucl. Instrum. Meth. A 506 (2003) 250.

[9] G. D’Agostini, "A Multidimensional unfolding method based on Bayes' theorem", Nucl. Instrum. Meth. A 362 (1995) 487.

[10] CMS collaboration, "Forward-backward asymmetry of Drell-Yan lepton pairs in pp collisions at $\sqrt{s}=$ 8 TeV", Eur. Phys. J. C 76 (2016) 325.

[11] ATLAS collaboration, "Measurement of the forward-backward asymmetry of electron and muon pair-production in $p p$ collisions at $\sqrt{s}=7 \mathrm{TeV}$ with the ATLAS detector", JHEP 09 (2015) 049. 